\title{
Retraction Note to: Aneuploidy analysis of non-pronuclear embryos from IVF with use of array CGH: a case report
}

\author{
Deng Lixin ${ }^{1,2} \cdot$ Xiang Zhifeng ${ }^{2,3} \cdot$ He Cong ${ }^{1} \cdot$ Zhang Jinzhou $^{2} \cdot$ Xie Hongbin $^{2}$
}

Published online: 28 December 2015

(C) Springer Science+Business Media Dordrecht 2015

\section{Retraction Note to: J Mol Hist (2014) 45:269-274 DOI 10.1007/s10735-013-9556-x}

This article has been retracted with approval of most of the authors, for the following reasons:

- The original article has already been published before in the Chinese language.
- The retracted article did not include all permissions needed.

- The retracted article contained errors.

The authors apologize for these mistakes and thank the reviewers of the journal.

The online version of the original article can be found under doi:10.1007/s10735-013-9556-x.

Xiang Zhifeng

zhifeng7809@qq.com

1 Animal Husbandry and Veterinary Institute of Henan Agricultural University, Zhengzhou 450002, Henan Province, China

2 College of Biology Science, Henan Institute of Science and Technology, Xinxiang 453003, Henan Province, China

3 College of Animal Science, Henan Institute of Science and Technology, Xinxiang 453003, Henan Province, China 